\title{
Standards en éducation, politique et professionnali- sation de l'enseignement: de la polysémie à la cacophonie? Questions (toujours) ouvertes
}

\section{Danièle Périsset Bagnoud}

La mise en cuvre du projet HarmoS et l'identification des "standards" y relatifs posent une série de problèmes théoriques, épistémologiques et pragmatiques qui sont loin d'être résolus. La présente contribution met en discussion certains de ces problèmes les plus importants: la question des relations entre politiques et chercheurs en éducation; la place respective que l'un et l'autre occupent ou sont appelés à occuper dans le domaine du pilotage du système éducatif; le problème de la définition des standards et de leur opérationnalisation, ou encore celui que pose l'interprétation polysémique des concepts de "professionnalisation" et "professionnels".

\section{Récurrences historiques}

En mai 2006, un nouvel article constitutionnel sur la formation est soumis au peuple. Accepté haut la main, il n’a pourtant pas été vraiment débattu, ni dans les milieux politiques, ni dans la presse, ni dans le champ scientifique. Tout s'est passé comme s'il était naturel de permettre à la Confédération de s'immiscer dans les affaires cantonales, du moins à propos de la formation. Il n'en a pas toujours été ainsi. Plus d'un siècle auparavant, le même objet avait donné lieu à d'intenses controverses. Lors de la consultation sur la Constitution fédérale du 29 mai 1874 , le fédéralisme helvétique et le poids des autonomies cantonales avaient eu raison des tendances centralisatrices suggérées par l'article constitutionnel relatif à la formation (Criblez, 1999; Périsset Bagnoud, 2003). Les questions relatives à l'instruction publique, à savoir les orientations gouvernementales et institutionnelles explicites qui engageaient les instances responsables, les cadres légaux et réglementaires ainsi que les ressources et moyens à mettre à disposition, ont suscité les réactions du monde politique qui les ont largement et vivement répercutées sur la place publique pour finalement les refuser. L'absence de débat tant de la part des politiques que de celle des professionnels en ce début $\mathrm{du} 21^{\mathrm{e}}$ siècle questionne, d'autant que la recherche en éducation, quasiment absente à la fin du $19^{\mathrm{e}}$ siècle, a acquis aujourd'hui des savoirs et des outils d'analyse 
permettant de décoder les enjeux et les finalités de tels objets et d'anticiper les difficultés et les conséquences des réformes envisagées. La recherche en éducation n'aurait-elle donc pas acquis de place légitime lui permettant de participer à la réflexion en amont et au débat publique relatif à cet enjeu de société que représente une réforme fondamentale du système éducatif?

\section{Un nouveau rôle dévolu à la recherche}

La recherche en éducation est pourtant bien présente dans les projets des politiques de l'éducation contemporains, mais la place qui lui est réservée n'est pas vraiment celle d'un pair, mais plutôt celle d'un mandataire dans un rapport de subordination hiérarchique. Par exemple, le rapport OCDE-CERI sur l'«Examen de la recherche et développement en éducation, Suisse, 2007» insiste sur la nécessité de développer une politique stratégique nationale et se réjouit de ce que le nouvel article constitutionnel sur la formation de mai 2006 le permette désormais. La recherche fondamentale resterait dans les seules universités (où les titulaires de chaires et responsables d'équipe pourraient continuer à mener des projets de recherche indépendante); la recherche appliquée serait à développer au sein des Hautes écoles pédagogiques où de nouveaux centres de recherche appliquée et de développement, financés sur concours et autour de projets dits "pertinents» (c'est-à-dire centrés sur leur mission principale et sur l'évaluation de leurs propres pratiques), seraient à créer. Les deux types d'institutions (universités et hautes écoles), complémentaires, instaureraient par ailleurs d'étroites collaborations. Il s'agirait de rassembler la coordination, la gestion, l'évaluation systématique et l'internationalisation des projets sous la responsabilité de la CORECHED $^{1}$ elle-même sous contrat avec la Confédération et les cantons. Dans ce contexte, les chercheurs devraient adopter une nouvelle posture:

Plus globalement, il convient d'encourager un sentiment d'autonomie afin d'obtenir plus de responsabilité et de réactivité dans le secteur de la recherche, ce qui lui permettra de jouer un rôle plus important et mieux coordonné dans le dialogue avec les dirigeants concernant l'élaboration des programmes de recherche ( $p$. 30).

De fait, donnant suite à l'amendement constitutionnel sur la formation, la mise en œuvre du projet HarmoS, prend cette direction. Une place y est bel et bien dévolue à la recherche, mais en aval: sur mandat de la Conférence des directeurs de l'instruction publique (CDIP), un consortium composé de scientifiques de plusieurs institutions romandes, tessinoises et alémaniques ${ }^{2}$ travaillent à rendre opérationnels, à l'horizon de l'automne 2008, les «standards» des disciplines d'enseignement jugées essentielles pour la scolarité obligatoire ${ }^{3}$. Parallèlement, un projet de «monitorage du système éducatif suisse» et un autre relatif aux «formations à l'enseignement en Suisse» sont formalisés. La récolte des données nécessaires à leur bon déroulement est confiée aux offices de récolte statistique 
(dont principalement l'OFS 4 ), aux services cantonaux de recherche mais aussi aux secteurs Recherche et développement des Hautes écoles pédagogiques (Wolter, 2006; Lehmann, Criblez, Guldimann, Fuchs \& Périsset Bagnoud, 2007; Maradan, 2006), tous instituts dotés dans ce cadre de mandats spécifiques coordonnés à un niveau supérieur, à savoir à un niveau politique. Les données récoltées dans le cadre des «monitorages» soutiendront le processus de pilotage et fonderont les prises de décision. Défini en tant que processus directeur, le monitorage réalisé dans chaque domaine permettra une évaluation itérative du système selon un principe hiérarchisé, conçu dans une logique «top-down» à laquelle seront invités à participer tous les acteurs, chacun à leur niveau:

Dans ce contexte, il serait particulièrement intéressant de savoir notamment comment des décisions prises à un échelon supérieur peuvent influencer les décisions des acteurs se situant à des échelons inférieurs du système éducatif. Le pilotage repose ainsi sur nombre de conditions préalables: il vise des objectifs et suit une orientation prédéfinie, il dépend d'acteurs à même de prendre des décisions et qui ont la compétence de le faire tout en disposant des instruments requis (compétence décisionnelle, compétence de réglementer, ressources appropriées, etc.). Dans ce sens, le pilotage [...] désigne l'influence ciblée exercée par des acteurs d'un niveau supérieur sur des acteurs subordonnés, afin d'amener ces derniers à modifier les structures et les processus de leur institution, de telle sorte que les indicateurs définis évoluent dans la direction souhaitées. Le pilotage est alors un processus auquel prennent part, de diverses manières, divers acteurs de différents niveaux du système éducatif (Lehmann et al., 2007, p. 116).

Politiques, administrateurs, chercheurs et enseignants, tous acteurs du champ éducatif, auraient dès lors une partition à jouer, partition définie d'en haut au service du pilotage du système, de son écologie, de sa rationalité économique. Pourtant, les acteurs ne partagent pas les mêmes enjeux ni ne poursuivent les mêmes finalités, en particulier en ce qui concerne la recherche en éducation.

\section{Recherche et discours politique}

Les chercheurs, issus essentiellement de la tradition universitaire, agissent sur la base de leur culture académique, à savoir avec une liberté certaine par rapport aux objets sur lesquels ils choisissent de se pencher et de porter leurs analyses, soutenues par un appareillage empirique qualitatif ou quantitatif. L'exercice de la distance critique, propre à leur statut académique, les rend prudents par rapport aux demandes politiques de "prestations de services" notamment en termes d'évaluation du système. D'un autre côté, les politiques ont, pour eux, la légitimité du pouvoir économique (par le financement des universités et des programmes de recherche) et celle, plus symbolique, du suffrage universel. Et lorsque cette dernière s'essouffle, c'est le jeu de la globalisation des politiques éducatives 
encouragées par les programmes d'évaluation et par «la vogue des classifications internationales (Benchmarking)»(Draelants, 2007, p. 7) qui la soutient, renforçant dans le même temps la légitimité économique du pouvoir politique. La pédagogisation du politique articule alors registre expert et sens commun; les décisions sont prises sur la base de «connaissances scientifiques» qui, loin de dépolitiser le débat, le re-politisent systématiquement par des échanges permettant aux acteurs de justifier les décisions idéologiques par une rhétorique où les faits ne sont plus lus dans leur réalité scientifique, mais selon les règles propres au registre et en relation étroite avec les enjeux politiques débattus (Draelants, 2007). L'usage social du concept de «standard» préoccupe le monde politique à ce niveau. Il délègue les défis de l'opérationnalisation et l'évaluation des «standards» à la recherche et au développement, puis leur mise en œuvre effective et contrôlable, aux enseignants sur le terrain.

\section{De La nature des "standards", questions ouvertes}

Mais, comme pour le concept de «compétence» (Crahay \& Detheux, 2005), celui de «standard» résiste et ne se laisse pas opérationnaliser si aisément. D'ailleurs, qu' est-ce qu'un standard? Est-ce une «super compétence», ou, au contraire, les standards représentent-ils des facettes observables et mesurables des compétences auxquelles ils sont naturellement subordonnés? Quels sont alors leurs éléments constitutifs? Si, dans le vocabulaire anglo-saxon le concept fait partie d'une culture partagée où les acteurs l'utilisent comme s'ils savaient exactement de quoi il est question et ne s'arrêtent plus pour rendre visible, clarifier et expliquer leur acception du concept de «standard», il en va différemment lorsque celui-ci est transposé dans la culture francophone. La question devient alors intéressante, parce qu'elle va chercher l'explicitation, l'identification de la fonction, du rôle, de la place des standards dans le champ conceptuel et dans celui de leur transposition pragmatique. Évidemment, la traduction littérale n'est jamais satisfaisante: les «standards» se traduisent-ils vraiment par «normes»? Mais alors comment expliciter, déplier, déployer une norme qui n'est pas celle du sens commun mais bien celle d'un champ social construit? Quelle est la nature d'une norme dans le champ de l'éducation? Est-elle consensus social, et alors quel est son rapport avec les apprentissages effectifs des élèves? Est-elle observable, et à partir de quels indicateurs, sur le terrain, mais alors quels éléments retenir pour ne pas réduire l'action de l'enseignant à une simple causalité (l'enseignement prescrit) suivie d'un effet, celui de l'apprentissage mesurable des élèves? Comment, dans ce cas, ne pas céder à la tentation néo-béhavioriste d'isoler les composantes de l'action de l'enseignant et celles (supposées) de l'apprentissage et nier leur complexité écologique, voire de la réduire à de trop simples (et vains) liens univoques de causalité, éloignant très certainement les objets évalués des caractéristiques des compétences auxquelles les standards sont pourtant étroitement liés? 
D'autre part, il reste encore à définir par quels processus pragmatiques l'introduction des «standards» et des procédures de rendre compte qui y sont liées accroissent réellement, si leur efficacité vient à être prouvée, les performances des élèves à long terme et dans le sens des compétences attendues. Y aura-t-il des effets agrégés que les travaux de recherche en éducation n'auraient pas permis d'anticiper, ou dont les résultats auront été négligés? Ainsi, plusieurs travaux récents établis à partir de vastes échantillons des États-Unis ou d'Angleterre ${ }^{6}$ observent les effets des standards sur la professionnalisation des enseignants. Ils constatent la fragmentation de leur identité professionnelle, la diminution de leur force de travail: les enseignants, dont l'épuisement et la démoralisation deviennent perceptibles, n'arrivent plus, ou avec difficulté, à reconstruire le sens pourtant nécessaire à tout processus de construction cognitive. Ils adoptent alors des attitudes de professionnalisation "managériale» plutôt que "démocratique» (Maroy, 2008). On peut poser l'hypothèse que ces attitudes managériales, qui ne s'occupent plus de l'atteinte des finalités globales de l'école pour viser avant tout, selon leur cahier des charges, l'efficacité et l'atteinte d'objectifs assignés par le politique et pour lesquels il est exigé de rendre compte, auront des effets sur les apprentissages des élèves, du moins si l'en en croit les travaux menés depuis longtemps déjà sur les "effets-enseignants» (Bressoux, 1994, 2001, Bressoux \& Bianco, 2004). Les savoirs accumulés par la recherche en éducation dans ce domaine permettent de poser des hypothèses fortes qui vont peut-être à l'encontre du projet politique à court terme: seront-elles entendues, en sera-t-il tenu compte, et comment?

Cette dernière question pose le problème sensible de la place de la recherche en éducation (en tant que science autonome) et des savoirs construits (à partir de preuves empiriques) dans ce chantier politico-social. Les chercheurs y ont-ils une place légitime et reconnue? Doivent-ils se battre pour la conquérir, ou la leur estelle laissée du bout des lèvres, ou sont-ils simplement exclus du débat, réduits à la position peu valorisée de fournisseurs de données, d'exécuteurs de mandats non négociés?

\section{Les modèles de la «professionnalisation»}

Une autre question, sous-jacente, émerge du débat sur les standards: celle de la professionnalisation. Leitmotiv des déclarations des politiques et des gens du terrain depuis la fin des années 1980 , le concept de professionnalisation fascine. Il est omniprésent en Suisse lorsque sont initiées les transformations des institutions de formation à l'enseignement et que les Hautes écoles pédagogiques remplacent les Écoles normales. Mais l'utilisation non théorisée du concept renforce le sens commun qui l'habite et contribue à la banalisation et à la dissolution de sa force intrinsèque. Car les professions sont issues d'histoires sociales spécifiques, longues et contrastées (Bourdoncle, 1991, 1993; Lang, 1999); elles se reconnaissent 
à plusieurs caractéristiques qui en font l'exigence mais aussi la valeur. L'enseignant professionnel, rappelle Tardif (2007, p. 172)

est d'abord vu comme un expert des processus d'enseignement et d'apprentissage en classe; son expertise repose sur la mobilisation d'une base de connaissances et de compétences efficaces issues de la recherche empirique, notamment en psychologie de l'apprentissage et en sciences de l'éducation. [...] Cependant, cette expertise enseignante est professionnelle dans la mesure où elle s'accompagne: 1) d'une formation longue (de type universitaire ou tertiaire) de haut niveau intellectuel; 2) d'une éthique ou déontologie basée sur le respect des élèves et le souci d'accroître leurs apprentissages; 3) d'une autonomie accrue des enseignants, non seulement dans la gestion des conditions d'apprentissage et le travail en classe, mais aussi en ce qui concerne leur participation à la gestion collective de l'éducation, principalement au niveau de l'établissement; 4) d'une valorisation, en termes de salaire, de statut et de carrière, de cette expertise professionnelle; 5) d'un pouvoir de contrôle plus important des enseignants sur leurs activités professionnelles et le travail de leurs pairs, notamment via un ordre professionnel. Le mouvement de professionnalisation vise aussi à améliorer l'image de l'enseignement dans l'opinion publique, à accroître son prestige social, notamment afin de recruter les meilleurs éléments pour le renouvellement de la profession.

Est-ce vraiment à ce professionnel-là que s'adressent les autorités politiques dans le cadre de HarmoS et des formations à l'enseignement subséquentes (Maradan, 2006), dans le rapport OCDE-CERI 2007 (voir supra) ou celui du monitorage de l'enseignement (voir supra)? Le rapport à la hiérarchie et à l'autorité énoncé renvoit-il à ce type de professionnel responsable, autonome et auto-régulé? L'orientation prise n'est-elle pas plutôt, clairement, celle, épistémologiquement différente, du modèle de la navigation professionnelle (Le Boterf, 2006) qui insiste sur la production "des règlements et des normes dont une des finalités est bien de donner confiance aux clients ou aux patients» (pp. 15, 23s.) et se réfere aussi à la délégation faite au professionnel de gérer lui-même sa mobilité et son employabilité ce qui "transforme les fonctionnaires en "professionnels», c'est-àdire en fournisseurs de services à des usagers avec qui ils sont liés par une relation de confiance dont ils cherchent à satisfaire les besoins»? (Dubar, 2000, p. 115). Ces manières complexes de concevoir le "professionnel» sont-elles complémentaires ou sont-elles contradictoires? Quels sont leurs points communs, où sont leurs divergences, à quel moment du processus apparaissent-elles, que disent-elles de manière implicite? Quelles conceptions sont convoquées par les différents acteurs qui l'utilisent, pour quels enjeux professionnels, politiques ou sociaux (Périsset Bagnoud, 2008)?

Il faut tenir compte de ces aspects dissociés, en tension certaine, si l'on veut rendre compte de l'usage social différencié qui est fait des concepts de «professionnalisation» ou de "professionnalité»: il conviendrait à présent, pour les acteurs du terrain comme pour les chercheurs et les politiques, d'identifier claire- 
ment les caractéristiques convoquées lorsqu'il est question professionnalisation dans le cadre de la mise en oeuvre de HarmoS, de standards, de recherche en éducation ou de pratique professionnelle. Cette identification conceptuelle permettrait de tirer, avec lucidité, les conclusions adéquates et d'adopter les postures cohérentes et efficaces par rapport aux finalités et stratégies des acteurs en présence qui, nécessairement, divergent d'une catégorie à l'autre.

\section{Ouvrir le débat et une réflexion constructive}

Telles sont finalement quelques unes des questions, épistémologiques, politiques et sociales, passionnantes et encore ouvertes auxquelles devraient notamment répondre, et dans un délai relativement court, les chercheurs et praticiens de l'éducation en charge d'opérationnaliser le projet HarmoS.

L'article engagé, voire polémique du prof. Walter Herzog, à la base de ce numéro «hors normes» de la Revue suisse des sciences de l'éducation, nous invite à entrer dans le débat sensible et stimulant des rapports potentiellement conflictuels entre les acquis de la recherche scientifique et les exigences des nouvelles politiques de l'éducation: espérons que le débat qui s'engage ici ne restera pas confiné à la section «chercheurs» de la noosphère mais que les diverses voix qui s'y expriment permettront d'établir un pont d'intelligibilité permettant de comprendre, d'être compris et de favoriser les liens entre les éléments disparates et parfois antagonistes que sont les logiques théoriques, pragmatiques et politiques.

\section{Notes}

1 Conférence suisse de coordination pour la recherche en éducation.

2 Pour la liste des institutions partenaires, se référer au document HarmoS, consortiums, juin 2005 disponible à l'adresse: http://www.edk.ch/f/CDIP/Geschaefte/framesets/mainHarmoS_f.html [23.01.2008]

3 À savoir: langue 1, langues étrangères, mathématiques, sciences naturelles.

4 Office fédéral des statistiques

5 Souligné dans le rapport original

6 Pour un compte-rendu des travaux récents de Ball, Osborne et Hargreaves à ce propos, voir Maroy, 2008.

\section{Références bibliographiques}

Bourdoncle, R. (1991). La professionnalisation des enseignants. Analyses anglaises et américaines. 1. La fascination des professions. Revue Française de Pédagogie 94, 73-92.

Bourdoncle, R. (1993). La professionnalisation des enseignants. Analyses anglaises et américaines. 2. Les limites d'un mythe. Revue Française de Pédagogie, 105, 83-119.

Bressoux, P. (1994). Les recherches sur les effets-écoles et les effets-maîtres. Revue Française de Pédagogie, 108, 91-137.

Bressoux, P. (2001). Réflexions sur l'effet-maître et l'étude des pratiques enseignantes. Les Dossiers des Sciences de l'Éducation, 5, 35-52 
Bressoux, P. \& Bianco, M. (2004). Long-term teacher effects on pupils' learning gains. Oxford Review of Education, 30 (3), 327-345.

Crahay, M. \& Detheux, M. (2005). L'évaluation des compétences, une entreprise impossible? (Résolution de problèmes complexes et maîtrise de procédures mathématiques). Mesure et évaluation en éducation, 28 (1), 57-78.

Criblez, L. (1999). L'article sur la formation dans la constitution fédérale du 29 mai 1874. In R. Hofstetter, Ch. Magnin, L. Criblez, \& C. Jenzer (Éd.), Une école pour la démocratie. Naissance et développement de l'école d'État démocratique en Suisse, 19 siècle (pp. 263-285). Berne: Peter Lang, Exploration.

Criblez, L., Hofstetter, R. \& Périsset Bagnoud, D. (Éd.). (2000). La formation des enseignant(e)s: Histoire et réformes actuelles. Berne: Lang.

Draelants, H. (2007). Les savoirs pédagogiques comme source de légitimation pour l'action publique en éducation. (Les cahiers de Recherche en éducation et formation, $\mathrm{n}^{\circ} 59,29$ pages). Louvain: Girsef et Chaire Unesco de pédagogie universitaire.

Dubar, C. (2000). La crise des identités: l'interprétation d'une mutation. Paris: PUF.

Lang, V. (1999). La professionnalisation des enseignants. Sens et enjeux d'une politique institutionnelle. Paris: PUF.

Le Boterf, G. (2006). Professionnaliser, le modèle de la navigation professionnelle. Paris: Organisation.

Lehmann, L., Criblez, L. Guldimann, T. Fuchs, W. \& Périsset Bagnoud, D. (2007). Les formations à l'enseignement en Suisse. Rapport dans le cadre du monitorage de l'éducation 2006. Aarau: CSRE/SKBF.

Maradan, O. (2006). L'enseignant suisse confronté à l'attente d'harmonisation et de résultats: contexte général et perspectives pour les institutions de formation. In D. Périsset Bagnoud \& É. Pagnossin (Éd.), L'enseignement aujourd'hui: contextes socio-politiques Formations et pratiques d'enseignement en questions. (Revue des HEP de Suisse romande et du Tessin, N5, pp. 133-151). Neuchâtel: CDHEP/IRDP.

Maroy, C. (2008). Régulation post-bureaucratique des systèmes d'enseignement et travail enseignant. Conférence au 20e colloque de l'ADMEE-Europe, Genève, Université, 9-11 janvier 2008.

Périsset Bagnoud, D. (2003). Vocation: régent, institutrice. Jeux et enjeux autour de la formation des enseignants du Valais romand, 1846-1996. Sion: Archives cantonales, Cahier Vallesia $\mathrm{n}^{\circ}$ 10.

Périsset Bagnoud, D. (2008). L'évaluation institutionnelle: discours d'intention ou étape du processus de professionnalisation? In C. Leclerc, La méta-évaluation: entre rationalité instrumentale et complexité. Genève: Université, actes [en ligne] du 20 ème colloque de l'ADMEE-Europe, 9-11 janvier 2008.

Wolter, S. (Éd.). (2006). L'éducation en Suisse. Rapport. Aarau: CSRE/SKBF.

Mots clés: Standards, HarmoS, compétences, professionnalisation, recherche en éducation 